\title{
ALGUNAS BASES EPIDEMIOLOGICAS DE LA MORTALIDAD INFANTIL
}

\author{
Dr. Alfredo Aguirre
}

\section{INTRODUCCION :}

Las observaciones a que nos referiremos en el presente trabajo son el resultado del análisis de las primeras 100 parejas pertenecientes al Programa de Planificación Familiar de la población semi-rural de Candelaria y representan el $12.8 \%$ de las mujeres expuestas a embarazo 0 sea de aquellas que hacen vida marital ya sea en matrimonio legal o en unión libre y entre los 15 y 44 años de edad.

También resume algunas de las observaciones efectuadas durante 20 meses de contacto con esta comunidad hechas a través de estudios socio-económicos y nutricionales.

Este análisis comprende el estudio de las repercusiones más importantes que, sobre la comunidad en general y la familia en particular, están teniendo las estructuras socioculturales y demográficas del país. También se presentan algunos resultados sobre como la familia trata de enfrentarse a ellas, aún con el cambio de sus actitudes hacia patrones culturales, sociales y religiosos, lo cual constituye un signo de alarma, ya que no sabemos las consecuencias que esto pueda tener sobre el núcleo familiar. Nos queda la duda de si eventualmente una de estas conse- cuencias no podría ser la desintegración de la familia.

También quedan analizadas en este artículo algunas de las bases epidemiológicas de la mortalidad infantil en nuestras comunidades y el papel que juega el médico como elemento fundamental en el origen y en la solución de los problemas que aquejan a la comunidad y a la familia.

\section{Estructuras Demográficas}

El problema de población en lo que a Colombia se refiere puede resumirse en la siguiente frase: "Cada 23 años o menos la población de Colombia se está duplicando".

Los siguientes datos nos demuestran que este hecho es un problema nuevo, por lo cual solo en los últimos años se le está dando la importancia que debe tener; así tenemos que:

1. Al descubrimiento de América, la población de Colombia se calcula en 850.000 habitantes (1492).

2. En el año de 1835 esta población llegaba a 1'686.038 o sea en 343 añns se había duplicado (primera duplicación en 343 años).

3. En 1905 esta cifra se elevaba a 4'300.000 habitantes (segunda duplicación en menos de 70 años). 
4. El censo de 1938 arrojó la cifra de 8'700.000 habitantes (tercera duplicación en 33 años).

5. Los cálculos para el censo de 1964 son de 17-18 millones (cuarta duplicación en 26 años).

6. La quinta duplicación se hará antes de 23 años o sea hacia 1987, la población será de 34-36 millones o más. (1)

Si lo anterior es fantástico por sus implicaciones sociales y económicas - ya que estos 34 millones de personas que estarán poblando a Colombia dentro de 23 años son seres humanos a los cuales hay que proveer de alimento, educación, vivienda, salud, vestido, etc.- el dato que arrojan investigaciones en algunas zonas rurales de Colombia y que muestran que la población se está duplicando actualmente no cada 26 años sino cada 16, hacen pensar que el problema no solo es fantástico sino que escapa a toda apreciación en sus consecuencias.

Las causas de este aumento de población son: la disociación que en los últimos años han tenido los índices de natalidad (los cuales han permanecido estables o ligeramente disminuídos), y los índices de mortalidad que han estado bajando continuamente en los últimos años (2). Esta disminución de mortalidad se debe, como en los demás países del mundo, a una aplicación progresiva de medidas de Medicina Preventiva y Salud Pública - vacunación - erradicación de enfermedades transmisibles uso de insecticidas y antibióticos etc.

\section{Esrtucturas Socio-Económicas}

De otro lado, el desarrollo económico del país apenas llega al $4.2 \%$ anual, lo que solo alcanza para sos- tener el actual nivel de vida, el cual es muy bajo. Las causas de este atraso en el desarrollo económico son múltiples, y entre las principales tenemos: distribución deficiente del ingreso nacional (el $20 \%$ de las familias colombianas reciben el $60 \%$ del ingreso) (3); técnicas deficientes en la explotación de la tierra; existencia de grandes latifundios y a su lado los minifundios; (el $64.2 \%$ de la superficie agrícola pertenece a $3.6 \%$ de los propietarios, mientras que el $4.2 \%$ de la superficie debe ser cultivada por el $56 \%$ de ellos) (4); luchas políticas por el poder que agravan los problemas sociales y la fuga de capitales hacia el extranjero.

\section{Repercusiones en la Comunidad}

El desequilibrio entre el crecimiento demográfico exagerado y un desarrollo económico deficiente se refleja: en un bajo ingreso per-cápita el cual llega a ser 10 y 15 veces menor que en otros países; aumento constante en el costo de la vida, 190\% en los últimos 10 años (5); desempleo ascendente, cada año ingresan 190.000 hombres a la masa trabajadora y de los cuales el $75 \%$ son analfabetas o solo han hecho uno o dos años de primaria y para los cuales no existe empleo (6); déficit de vi-

(1) Juan Luis de Lannoy - Gustavo Pérez "Estructuras Demográficas y Sociales de Colombia". Centro de Investigaciones Sociales. Bogotá - 1961.

(2) R. Albert Berry "Breve Estudio de los determinantes del Crecimiento de la Población en Colombia" - Universidad de los Andes: Centro de Estudios sobre Desarrollo Económico. 1964.

(3) José Lebret "Estudio de las condiciones de desarrollo en Colombia". Bogotá 1958.

(4) Gustavo Pérez "El Campesinado Colombiano" Bogotá. Centro de Investigaciones Sociales.

(5) DANE "Boletín Mensual de Estadística". Agosto 1965.

(6) SENA "La Formación Profesional y el Desarrollo Económico y Social”. Bogotá, 1964. 
vienda (800.000) a los cuales se agregan 50.000 cada año (7). En el campo social las repercusiones no son menos graves: la educación es deficiente (solo el $20 \%$ de los colombianos terminan escuela primaria $y$ el $3 \%$ secundaria) ( 8 ); en los servicios de salud su impacto es notorio: hay un médico por 2.400 habitantes y una enfermera por 16.000 (9). A los hospitales llega un exceso de pacientes a los cuales no es posible atender adecuadamente, así que toda la atención está dedicada a la medicina curativa y poco a la preventiva, creando el círculo vicioso de: poca prevención $=$ más enfermedad = menos prevención. Lo anterior parece confirmarse al analizar nuestras cstadísticas de mortalidad infantil y de morbilidad encontrándonos que la desnutrición llega al $40 \%$ en algunas áreas y la diarrea pasa del $30 \%$ en nuestras comunidades infantiles (10). Estas dos entidades constituyen los pilares donde se asiontan las causas de nuestra mortalidad infantil.

En cuanto a los servicios públicos la situación no es menos precaria: según el censo de 1951 el $50.6 \%$ de las viviendas urbanas carecían de baño, el $31.1 \%$ sin agua, el $35.7 \%$ sin luz y el $25.7 \%$ con piso de tierra; en la vivienda rural la situación es aún más dramática, el $92.6 \%$ sin acueducto y el $88.7 \%$ no tiene sanitario. En cuanto a los demás servicios sociales: protección del niño abandonado y del anciano es poco lo que cxiste a pesar de que este tipo de población está en aumento constante.

En cl aspecto social cada día es más irrecuente y grave entre nosotros los motines, cierre de fábricas, huelgas, despiclo de trabajadores, violencia, invasiones todo lo cual es reflejo de la situación.

\section{Repercusiones en la estructuración $y$ situación en que vive la familia}

Otro aspecto y quizá el más importante de analizar es el impacto en la salud y el intelecto de la familia colombiana. Para mejor entender este punto analicemos la forma como se estructuran y la situación en que vive gran parte de las familias residentes en la población semi-rural colombiana de Candelaria, donde se han llevado a cabo estudios socio-económicos, nutricionales y de actitudes hacia la planificación familiar; los hallazgos revelan los siguientes hechos:

La familia en promedio está constituída de 6.7 miembros el $25 \%$ son analfabetas y del resto solo el 10\% han terminado escuela primaria $y$ ninguno secundaria reduciéndose sus conocimientos de escritura y lectura a saber contar en cifras no muy complicadas y su vocabulario de lectura a unas pocas palabras. En cuanto a su constitución familiar, revelan un patrón de transición entre los litorales y zonas mineras donde prevalece la unión libre y el interior del país, donde ésta es muy baja (11); en nuestro caso encontramos un $44 \%$ de uniones libres: el $35 \%$ de las mujeres habían tenido uno o más hijos antes de la actual unión marital (con una alta mortalidad entre

(7) Naciones Unidas "Estudio Económico de América Latina". 1963.

(8) DANE. "Anuario General de Estadística". 1958.

(9) Asociación Colombiana de Facultades de Medicina. "Estudio sobre Recursos Humanos". En estudio. 1964.

(10) Wray - Aguirre. "Estudio de la Prevalencia de diarrea y desnutrición en Candelaria". 1964.

(11) Virginia Gutiérrez de Pineda: "La Familia en Colombia". Centro de Investigaciones Sociales. Bogotá. 1962. 
cllos); sus primeras relaciones sexuales habian tenido lugar en el $28.5 \%$ antes de los 15 años y en el $70 \%$ antes de los 18 años la edad promedio de la madre 30 años y el tiempo que llevaba en la última unión marital 10 años.

Los datos anteriores dejan clara la idea de como se constituyen estos matrimonios, y así tenemos que la mujer usualmente analfabeta o semianalfabeta, desde muy temprano inicia sus relaciones sexuales en forma esporádica, pero de las cuales nacen hijos, que a la postre vienen a constituirse en un estorbo para la madre, ya que no posee recursos económicos con que satisfacer sus necesidades.

Ante esta situación solo le quedan dos soluciones: la primera es buscar ya no solamente por instinto sexual sino por necesidad económica, una unión más o menos estable, aún a expensas de sacrificar su propia libertad, es decir, en este tipo de unión el hombre es el que decide el tiempo que durará ésta, distribuirá el presupuesto familiar cle acuerdo a sus conveniencias (otras mujeres, alcohol, etc.) y determinará el número de hijos, etc. La otra manera de solucionar sus problemas es con la muerte del niño (infanticidio encubierto), en estos casos es frecuente observar como infantes entre seis meses y cuatro años son abandonados a su suerte cuando cualquier enfermedad los ataca, especialmente diarrea, y así, encontramos madres que se oponen a que sus hijos sean tratados o contrariadas cuando el tratamiento ha sido exitoso. En otros casos no menos raros, estos infantes son abandonados en los hospitales, constituyendo un problema difícil de solucionar. También refleja este tipo de solución, que a sus problemas da la madre o pareja de bajos recursos, cuando se acerca al médico a pedir la "boleta de defunción" de su hijo, sin llegar a manifestar ningún sentimiento de angustia lo cual deja al médico tan desconcertado que no acierta a explicar que está pasando en aquella madre.

En cuanto a la situación económica de estas familias, no puede ser peor. El ingreso promedio mensual es de $\$ 400,00$ a $\$ 500,00$ (US $\$ 40,00$ US $\$ 50,00$ dólares al cambio oficial) por familia, los que deben ser repartidos en alimentación, vivienda, vestido y educación. Ante esta situación de pocos recursos económicos $y$ la urgencia de satisfacer necesidades biológicas, la familia se decide por satisfacer la más elemental, como es la de comer, así que su presupuesto es dedicado en su mayor parte a comprar alimentos el $98.7 \%$ de las familias gastan más del $40 \%$ en comida (12); ahora bien, estos alimentos para poder calmar en algo el hambre (estómago lleno), dében ser voluminosos y baratos (carbohidratos).

Esta situación se va agudizando con el paso de los años, a medida que el costo de la vida aumenta y el número de hijos crece, todo esto sin que haya una compensación manifestada en mayores ingresos para la familia, ya que aunque el jefe de la casa se va envejeciendo esto no representa mayores ingresos que compensen los otros factores (alto costo de la vida y mayor número de hijos). (12)

En este momento, la alternativa es dedicar aún más porcentaje del presupuesto a comida, encontrándose por este tiempo familias (con cuatro - más hijos) que están dedicando el

(12) Wray - Aguirre. "Estudios Epidemioló gicos sobre Desnutrición en Candelaria". 1965. 
$60 \%$ o más, para satisfacer esta neciedad, no puede ser otra que de vienda, vestido y educación no pueden ser atendidas.

\section{Repercusiones en el individuo}

La situación en este momento es dramática para aquellas familias con hijos numerosos y las encontramos tratando cle acomodar en una o dos piezas a seis o siete personas, con cscaso vestido, analfabetas y como la comida es poca y clebe ser dada en su mayor parte al jefe de la familia "porque es el que trabaja", queda una poca cantidad para los hijos, los cuales desde la vida intrauterina (17\% de Prematurez) (13) empiezan a padecer del fenómeno del hambre y los factores pobreza, ignorancia, enfermedad comienzan a dejar en ellos sus huellas, dándonos como resultado un niño colombiano desnutrido.

Esta desnutrición posteriormente será la causa de su muerte (14) y si esto no ocurre dejará funestas consecuencias en su desarrollo psico-motor $(15,16)$ los cuales se manifestarán primero en falta de habilidad en sus movimientos, retardo en caminar, atraso en la iniciación de su lenguaje, dificultades para relacionarse con las personas y con una capacidad de adaptación a las diferentes circunstancias del ambiente disminuída. Lo anterior ocurre entre el nacimiento y los cuatro años.

Posteriormente en la escuela su rendimiento intelectual es bajo, conflictivo con los profesores, y por último la abandona y así, sin ninguna capacitación, consecuencia de su desnutrición crónica y de la falta de oportunidades, ingresa a la masa de los desocupados, constituyéndose en un problema social.
En otros casos logra ocupar un trabajo, el cual cumple siguiendo las mismas características generales de bajo rendimiento y conflictivo con sus patrones.

En cuanto a su actuación en la sociedad, no puede ser otra que de antagónicss con la autoridad y de desadaptación ante una sociedad que los rige con leyes y normas que no conocen, porque nadie se las ha enseñado y que les nieja el derecho a la supervivencia a ellos y a su familia. Esta situación es la que se agudiza cada clía más y más en nuestras ciuciades y que se manifiesta en los frecuentas motines, desórdenes callejeros, invasiones de tierras, violencia en los campos, etc.

Analizada la forma como se estructuran cstas familias y la situación en que viven, vale la pena preguntarnos sobre cuál es el efecto que la situación anterior está produciendo en la familia colombiana que se ve acosada por el hambre, la miseria y la ignorancia, fenómenos estos agudizados por la presencia de más hijos, a los cuales no se les puede satisfacer ni siquiera en sus necesidades biológicas como son las de comer, vestirse, recibir educación y tener un hogar cloncle vivir y que está llevando a situaciones como las descritas, en que madres, consciente 0 inconscientemente, dejan morir a sus hijos de pocos años.

(13) Alfredo Aguirre. "Estudio de Prematurez en Candelaria". 1964.

(14) Pelayo Correa. "Estudios sobre Defunciones del niño en Colombia". Antioquia Médica. 9:54, 1959.

(15) Ramos Galván R. y Cravioto J. "Desnutrición en el niño". Bol. Médico. Hospital Infantil de México. 15:763-788. 1958.

(16) Robles B. Ramos Goluan R. y Cravioto J. "Valoración de la conducta del niño con desnutrición avanzada y de sus modificaciones durante la recuperación". Bol. Méd. Hospital Infantil de México. 


\section{El Médico como factor contribuyente al problema. Papel del Médico}

Antes de tratar de dar una respuesta analicemos un poco cual ha sido la contribución del médico a esta situación. Para mejor analizarlo veamos cuál ha sido su entrenamiento: Al médico siempre se le ha enseñado que debe evitar que el enfermo se muera y en su labor se ha visto completamentado por los descubrimientos de drogas eficaces contra las enfermedades y las plagas, pudiéndose decir que hasta el presente ha cumplido su labor de evitar que el enfermo se muera. Pero ha descuidado en sus planteamientos otros componentes no menos importantes de lo que es SALUD, la cual se define como el completo bienestar físico, mental y social del individuo (17) y para lo cual se entrena poco al médico. Si analizamos esta definición de salud y las consecuencias que para la familia está representando el tener hijos a los cuales no se les puede satisfacer sus necesidades biológicas y que están llegando a la edad adulta con secuelas en su esfera mental dejadas por la desnutrición y la falta de oportunidades en la escuela. Podemos ver entonces, como se está formando un círculo vicioso de menos muertos, menos bienestar físico, mental y social y podemos predecir que si se sigue agudizando este círculo, el médico perderá su función como preservador de la salud.

Su falla ha sido no participar en la planeación de programas de desarrollo económico y social como parte integral en los planes de salud. ¿Por qué no lo ha hecho? Quizás lo explique la orientación de su entrenamiento hacia curar enfermos y a no considerar que lograr el bienestar físico, mental y social en el individuo es su labor y lo cual solo alcanzará cuando conozca y participe en la planeación del desarrollo del país, para lo que debe prepararse, orientándose y documentándose en la Universidad sobre los problemas que aquejan la nación y la familia.

Ante la actual situación, qué debe hacer el médico y cuál debe ser su participación en el tratamiento de los problemas que aquejan a la familia?

\section{Repercusiones manifestadas en cambio de actitudes}

Tal vez quede clara la respuesta al observar las consecuencias que sobre la familia están teniendo sus "victorias". También servirán estas observaciones para dar contestación a la pregunta que nos habíamos formulado inicialmente sobre, qué estaba pasando en la familia que al no tener recursos económicos para satisfacer las necesidades biológicas de sus miembros y que al agudizarse el problema con el nacimiento de un nuevo hijo decide en algunos de los casos "sacrificar" a este nuevo miembro?

Estas observaciones dan los siguientes datos: el $84 \%$ de las mujeres habían tenido su último embarazo en los dos años anteriores, $74.2 \%$ tenían menos de una relación sexual por semana, $68.5 \%$ rara vez o nunca presentaban orgasmo en sus relaciones sexuales y el $78.7 \%$ consideraban un problema económico tener otro hijo.

De los anteriores datos podemos deducir que existe una alta fertilidad entre estas mujeres, ya que sin ser muy frecuentes sus relaciones sexuales y practicar métodos anticoncepti-

(17) Definición de la Crganización Mundial de la Salud. 16:317-341, 1959. 
vos ( $\sin$ técnica), es muy alta la frecuencia de embarazos.

En cuanto al poco número de relaciones sexuales y alta incidencia de frigidez, parecen tener su explicación cn el hecho frecuente de pensar que si tienen orgasmo pueden quedar embarazadas y son más buscadas por el hombre con más probabilidades de embarazo, el cual no quieren. Así que siendo frígidas logran espaciar las relaciones sexuales y "evitar" el embarazo que no quieren. En otras ocasiones este rechazo de la mujer al marido se hace abiertamente. Este último hecho es llamativo, si se tiene en cuenta que puede estar indicando un cambio de actitudes en la esfera sexual, en lo que se refiere al comportamiento sexual de la mujer frento al hombre.

La misma sensación me invadió cuando, antes de comenzar el programa de planificación familiar se repartieron boletas invitando a las mujeres a que ingresaran al programa pero con el requisito de que debían de traer dicha boleta firmada por el marido, encontrándonos que más del $50 \%$ de los maridos respondió afirmativamente, sin que nosotros hubiéramos tenido contacto directo con él.

Otros datos son: el $19.5 \%$ de las mujeres habian terminado su último embarazo en aborto 0 mortinato y el $53.8 \%$ reconocían haber practicado maniobras anticonceptivas; lo anterior, y teniendo en cuenta que el aborto (provocado), es la primera causa de hospitalización después del parto, hace pensar que la situación angustiosa en que vive la familia (hijos numerosos, bajos ingresos, hambre, ignorancia, enfermedad) está haciendo que como medida defensiva para conservar su integridad, recurra al aborto y a las prácticas anti- conceptivas cada vez con más frecuencia; estas últimas van desde las más primitivas hasta las más modernas, como son las tabletas orales y así tenemos que el coitus interruptus es usado en el $37.3 \%$ de las parejas, el condón en el $23 \%$, tabletas vaginales no efectivas $11 \%$, tabletas orales $9.9 \%$, método del ritmo $10 \%$, duchas $22 \%$.

Así que podemos ver como prácticamente usan todos los métodos conocidos pero sin ninguna técnica $y$ no de una manera regular. Lo anterior solo nos indica una cosa y es la necesidad que SIENTEN las parejas de limitar el número de sus hijos para lo cual están recurriendo a métodos aún tan drásticos como es el aborto y el infanticidio encubierto; csta necesidad también se manifiesta cuando al ofrecerles un programa de planificación familiar lo aceptan casi espontáneamente.

En cuanto a sus ideales de fecundidad: el $83.8 \%$ quisieran haber tenido tres hijos o menos. El $80 \%$ considera que entre los 18 y 22 años está la edad ideal para tener el primer hijo y el $75 \%$ que la edad ideal para tener el último está entre los 25 y 30 años. El $80.4 \%$ consideran como intervalo ideal entre los embarazos 2 a 3 años y 4 o más años el $20 \%$. Estos ideales son completamente antagónicos con su situación actual, lo que parece indicar un cambio de actitud hacia la composición de la familia.

Otros datos dignos de analizar es el papel que juega la Iglesia Católica en estas familias, al preguntar sobre prácticas religiosas (misa semanal o mensual y comunión anual) encontramos que el $44.5 \%$ solo concurren ocasionalmente o nunca a misa y el $35.8 \%$ nunca comulgan; al profundi- 
zar más en este punto encontramos las siguientes respuestas de las madres: "no volví a la Iglesia porque ya estoy condenada, ya que vivo en unión libre", si se les pregunta por qué usan prácticas anticonceptivas siendo prohibidas por la Iglesia dan una respuesta vaga como: "yo no se, pero no quiero tener más hijos".

Esto deja ver como el fenómeno de la sobrepoblación parece estar cambiando las actitudes de nuestras familias hacia la religión; podrían señalarse como causas de ello la disminución de la relación sacerdote por número de habitantes ya que las circunstancias actuales están haciendo que la familia se aleje más de las doctrinas de la Iglesia, para así poder solucionar sus problemas ya que su otra alternativa es perecer.

Lo anterior se confirma con las respuestas dadas al preguntárseles por qué no usan anticonceptivos de una manera regular: por falta de información $41 \%$, por temor a enfermar $30.4 \%$, vergüenza con el cónyuge $14.8 \%$, negligencia $4.7 \%$, temor a la Iglesia $4.7 \%$, sin dinero $1.6 \%$ porque lo usan mujeres malas $1.6 \%$, porque quieren hijos $1.6 \%$. Como vemos, solo un $4.7 \%$ respondieron que no usaban anticonceptivos por temor a la Iglesia. Es claro también que dos factores están contribuyendo a que la planificación familiar SENTIDA por las familias no sea efectiva, y éstas son: la falta de educación sexual y la falta de información técnica.

En resumen estas familias están haciendo la planificación a su manera, recurriendo a todos los métodos conocidos: primero hay rechazo de la mujer al marido en las relaciones sexuales, a veces consciente y abiertamente, en otras "inconscientemen- te $^{\prime \prime}$ y manifestada a través de frigiclez; luego recurre a los métodos anticonceptivos, pero usados con deficiencias técnicas, si esto falla, recurre al aborto o al "infanticidio encubierto". Como es de suponer, esta necesidad de limitar la familia obedece a leyes biológicas de supervivencia y cuando se llega a los extremos actuales, la familia debe cambiar algunos de sus principios morales, religiosos, culturales y de integración familiar, que se manifiestan en cambios de actitudes, pero cambios que por ser tan rápidos son traumáticos y difíciles de asimilar completamente y no sabemos cuales serán las consecuencias definitivas que sobre la familia pueda llegar a producir, quedando la duda de si no podrían llevarla a su propia desintegración.

Planteadas las posibles consecuencias que sobre la familia están teniendo los "éxitos del médico", parece claro el camino que le tocará seguir a éste en la búsqueda de la salud de la familia y del individuo y el cual no parece ser otro que el de mantenerse informado de todos aquellos factores que condicionan el bienestar físico, mental y social para guiarlos en su beneficio. En segundo lugar, estar atento a los llamados que como en las actuales circunstancias está haciendo la familia para acudir en su ayuda y guiarla hacia la solución de sus grandes problemas.

En esto es precisamente en lo que cstán empeñadas las directivas médicas del país, pero que no podrán cumplir si cada uno de los médicos no colabora participando activamente en la solución.

Pero no siempre el médico puede actuar con todas las armas que la ciencia le da y a veces encuentra li- 
mitaciones ya no solo de la naturaleza sino también sociales y religiosas que, aunque en la actualidad parecen difíciles de vencer, las mismas comunidades, ante la inminencia de su desintegración, se están encargando de romper, tal como parece estar ocurriendo hoy día.

\section{CONCLUSIONES}

Existe un problema demográfico colombiano el cual es nuevo y está agravado por las estructuras socioeconómicas y culturales que actualmente existen en el país.

Este problema se está manifestando en la comunidad a través de una recesidad sentida de planificar la familia la cual actualmente hace, pero a su modo y no de una manera efectiva encontrándonos como principal causa para no lograr su objetivo, la falta de educación sexual e información técnica.

Parece existir en la comunidad un cambio de actitudes hacia algunos de los patrones sociales, culturales, religiosos y de integración familiar, como consecuencia de las medidas que está adoptando para limitar la familia, quedándonos la duda de si estos cambios por ser tan rápidos no podrán llevarla a su propia desintegración.

También queda claro al analizar el problema y sus consecuencias en el niño colombiano, algunas de las bases fundamentales en la ejidemiología de la mortalidad infantil y de nuestra patología social.

En cuanto al papel del médico en la solución de uno de los grandes problemas que aqueja a la familia en Colombia: necesidad sentida de planificar la familia, la cual es el resultado de una presión demográfica familiar y de una estructura socioeconómica y cultural que no le ofrece garantías es acudir en su ayuda facilitándole los elementos necesarios como son educación sexual y asistencia técnica para así ayudarla a lograr su objetivo. De otro lado, participar más activamente en el desarrollo económico y social del país.

\section{RESUMEN}

Se hace una revisión del panorama demográfico, socio-económico y cultural de Colombia analizando sus repercusiones en la comunidad y la familia en Candelaria, encontrándose a través del análisis algunas de las bases epidemiológicas de la mortalidad infantil en nuestra comunidad. 